AIAA-2002-1529

\title{
BUCKLING TESTING AND ANALYSIS OF SPACE SHUTTLE SOLID ROCKET MOTOR CYLINDERS
}

\author{
Submitted to the \\ $43^{\text {rd }}$ AIAA/ASME/ASCE/AHS \\ Denver, $\mathrm{CO}$ \\ Structures, Structural Dynamics \& Material Conference \\ Thomas J. Weidner \\ ATK Thiokol Propulsion \\ A Division of ATK Aerospace Company \\ April 22-25, 2002 \\ David V. Larsen \\ ATK Thiokol Propulsion \\ A Division of ATK Aerospace Company
}

\begin{abstract}
A series of full-scale buckling tests were performed on the space shuttle Reusable Solid Rocket Motor (RSRM) cylinders. The tests were performed to determine the buckling capability of the cylinders and to provide data for analytical comparison. A nonlinear ANSYS Finite Element Analysis (FEA) model was used to represent and evaluate the testing. Analytical results demonstrated excellent correlation to test results, predicting the failure load within $5 \%$. The analytical value was on the conservative side, predicting a lower failure load than was applied to the test. The resulting study and analysis indicated the important parameters for FEA to accurately predict buckling failure. The resulting method was subsequently used to establish the pre-launch buckling capability of the space shuttle system.
\end{abstract}

\section{INTRODUCTION}

The RSRM used in the space shuttle launch system is made up of twelve thin wall steel cylindrical segments (see Figure 1). During pre-launch, launch and liftoff, the two RSRMs support the entire space shuttle liftoff vehicle. Bolts with nuts, that are severed by small explosives (frangible nuts), hold the aft end of the RSRMs fixed to the Mobile Launch Platform until just prior to liftoff. To ensure that the shuttle does not drift into any part of the Mobile Launch Platform, the space shuttle main engines are started approximately six seconds prior to liftoff. During the pre-launch phase of liftoff the space shuttle main engines produce approximately one million pounds of thrust. The RSRMs respond to this load by initially bending along the motor, resulting in a 26 inch displacement at the RSRM nosecone and then rebounding back to the original upright position. As soon as the vehicle has returned to the upright position, the frangible nuts are triggered, freeing the shuttle for liftoff with enough lateral inertia to ensure safe ascent without the possibility of impact on the Mobile Launch Platform.

The initial bending during pre-launch generates a significant structural load on the RSRMs. The severity of this loading condition is further complicated by the lack of internal pressure to support the thin wall of the RSRM cylindrical membrane. At this point during the launch, buckling becomes a significant concern. In order to avoid the possibility of a buckling event, several parameters (wind speed and direction, payload, membrane thickness and imperfection, etc.) are restricted, and stringent liftoff vehicle configuration requirements have been established. As liftoff requirements become more restrictive, the probability of buckling is reduced, however, the probability of a launch delay or cancellation is increased. One of the primary concerns is wind. High wind speed can significantly increase the horizontal load and the total bending load (see Figure 1). Therefore, wind speed and direction are closely monitored prior to lift-off.

Using a conservative linear eigenvalue analysis to determine the RSRM's buckling capability resulted in wind speed allowables more restrictive than necessary. As the space shuttle program has developed, several modifications have been incorporated to improve performance and efficiency. As part of an enhancement activity, significant effort has been invested in trying to decrease the probability of a launch abort or delay due to wind speed concerns.

It is a common practice in industry to use eigenvaluebuckling analysis to establish preliminary design parameters. The basic structure is analyzed using the 
eigenvalue approach. Large safety factors are used with applied loads and conservative knock down factors (KDF) are applied to the results. This ensures that the structure never approaches the buckling limit. It is

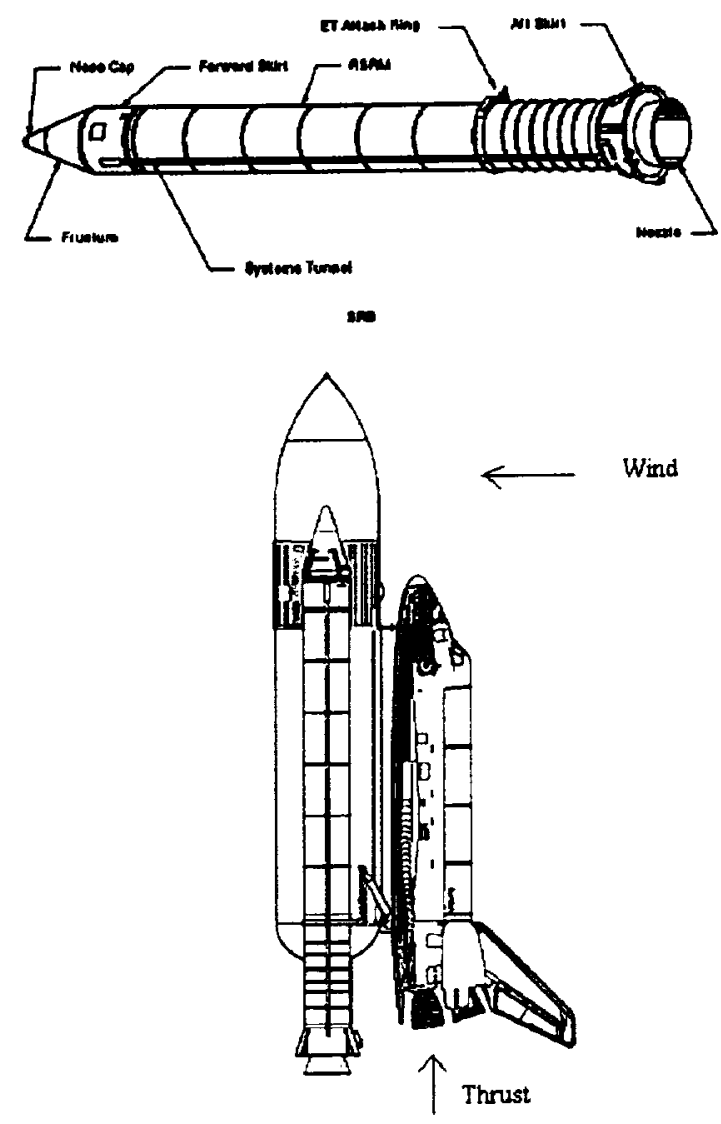

\section{Figure 1 Reusable Solid Rocket Motor and Space} Shuttle System

understood that a pure eigenvalue buckling analysis is not a precise representation of the actual buckling capability. Most experimental data uses small diameter, thin wall cylinder test specimens to determine the KDF. Little empirical data exists for buckling of cylindrical shells of this size. The use of small diameter thin wall cylindrical test data to formulate a KDF for large diameter cylinders resulted in the restriction of allowable flight wind speed and the establishment of stringent liftoff vehicle configuration requirements. To eliminate over-conservatism and increase the allowable wind speeds, improvement to the linear eigenvalue analysis to determine buckling capability was required.

A full-scale RSRM buckling test program was completed to develop and validate a nonlinear finite element analysis approach to determine buckling capability. Nonlinear analyses using the ANSYS finite element code were performed on the test arrangement in order to validate a detailed approach to predict buckling failure.

\section{DISCUSSION}

\section{Test Setup}

Testing of full-scale RSRM hardware began in the winter of 1998. The primary objective of the test program was to obtain empirical data to validate analytical models, which would then be used to predict the buckling capability of the RSRM case structure under the space shuttle launch vehicle pre-launch load environments. Additional objectives of the testing included the development of an accurate simulation of flight loading, generation of data to support the study of critical buckling variables, determination of actual capability of flight hardware by loading full scale hardware to failure, verification of the finite element model and accuracy of the nonlinear capabilities, and the verification of the nonlinear method for predicting buckling capability.

The buckling capability of a structure is highly dependent on the type of load applied. When performing a certification analysis, it is critical to duplicate the expected loading, eliminating the inaccuracy associated with a loading difference. Significant effort was invested in the design of a test fixture to closely approximate the pre-launch loading conditions of the space shuttle system on the RSRM.

During the critical buckling phase, pre-launch loading causes the axial stress in the RSRM to peak in the aft stiffener cylinder, which is the critical location for RSRM buckling. Each end of the cylinder transitions from membrane into a thicker section with the forward end being a clevis and the aft end a tang (see Figure 2). On subsequent cylinders the clevis and tangs are mated together using pins. The stiffener cylinder includes two integral stiffener stubs that divide the stiffener membrane into three sections or bays (forward, center, and aft). During flight, stiffener T-rings are bolted to the stiffener stubs to increase the strength of the cylinder during splashdown.

The test set-up (see Figure 3) was arranged to test a single RSRM cylinder loaded similarly to what an actual flight cylinder would experience during prelaunch of the space shuttle system. The test article consisted of an RSRM stiffener cylinder. Clevis and 


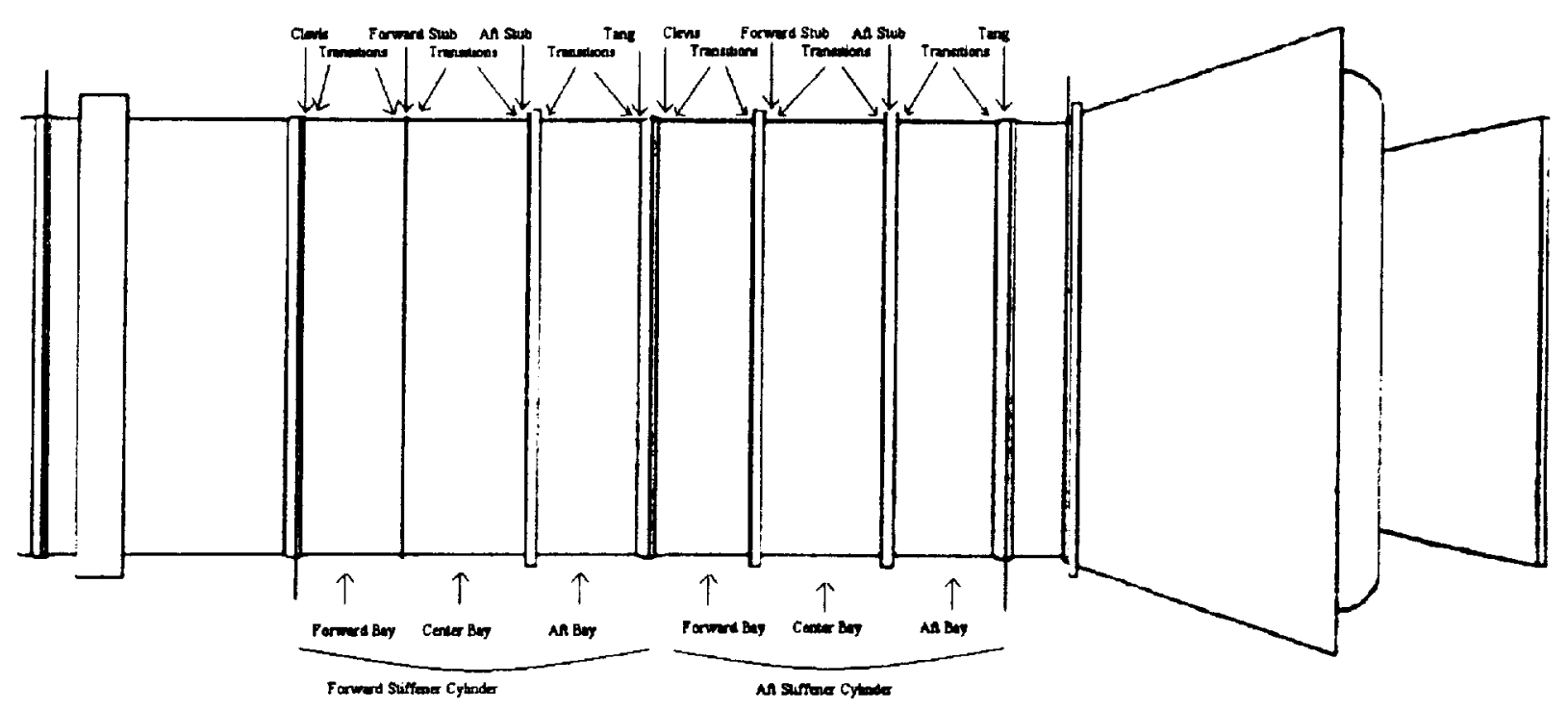

Figure 2 Stiffener Cylinder Nomenclatures

tang spool cylinders were pinned to either side of the stiffener cylinder to simulate flight joint constraints and ensure similar joint behavior. Adding the spools produced approximately the same bending stiffness as a full sized cylinder, allowing the joint and stiffener membrane to rotate and deflect similar to the flight configuration. Upper and lower buckling rings were attached to the spools.

The force beams applied the force to the buckling rings. Buckling beams transferred the load to the force beams. A force frame connected the buckling beams and was driven by six actuators that impose the load on the structure. In order to achieve loading similar to prelaunch flight loading, the force frame was located to represent the maximum expected load and closely approximate the correct load distribution in the RSRM stiffener.

Another important factor in the test was the accurate transfer of load from the test fixture to the test article. Much like the type of loading, boundary conditions or fixity of a structure can significantly influence the buckling capability. To avoid uncertainty, the test fixture was designed to simulate the RSRM pre-launch arrangement as closely as possible. An oversimplification of the end conditions, like a simply fixed interface, would introduce significant inaccuracy. Test stand height limitations prevented the use of full cylinders to simulate end conditions for the test cylinder. Therefore, analysis was performed to determine the required length (axial direction) of membrane that would allow proper joint rotation (stiffness) and overall joint behavior. The required membrane length was used to produce two spools to simulate the use of full cylinders (see Figure 3).

The test load was designed to be slightly different than the pre-launch load. The simple application of an axial load to a moment arm produces a load that varies in the

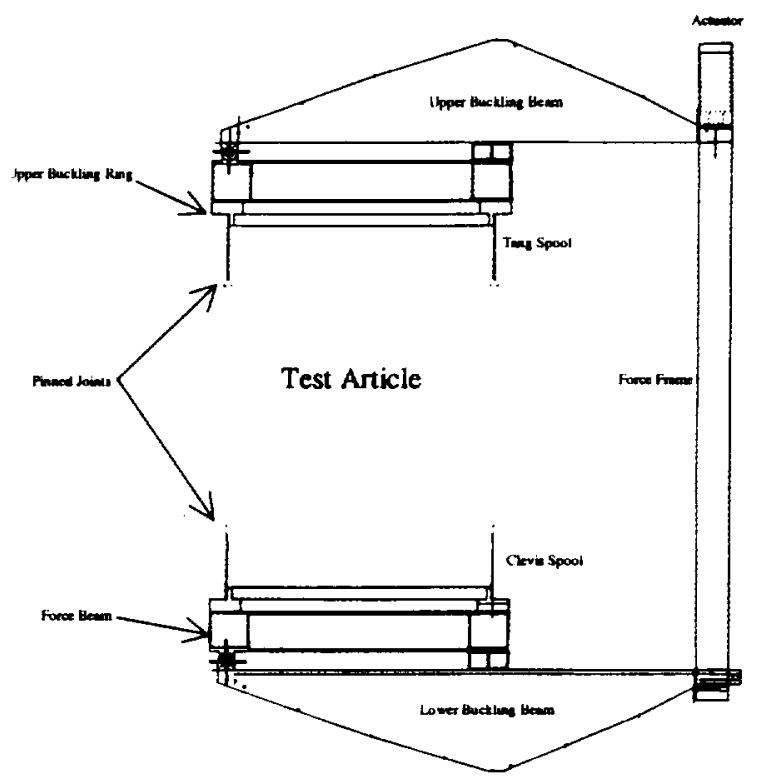

Figure 3 Buckling Test Set-up 


\section{Comparison Flight to Test Loading}

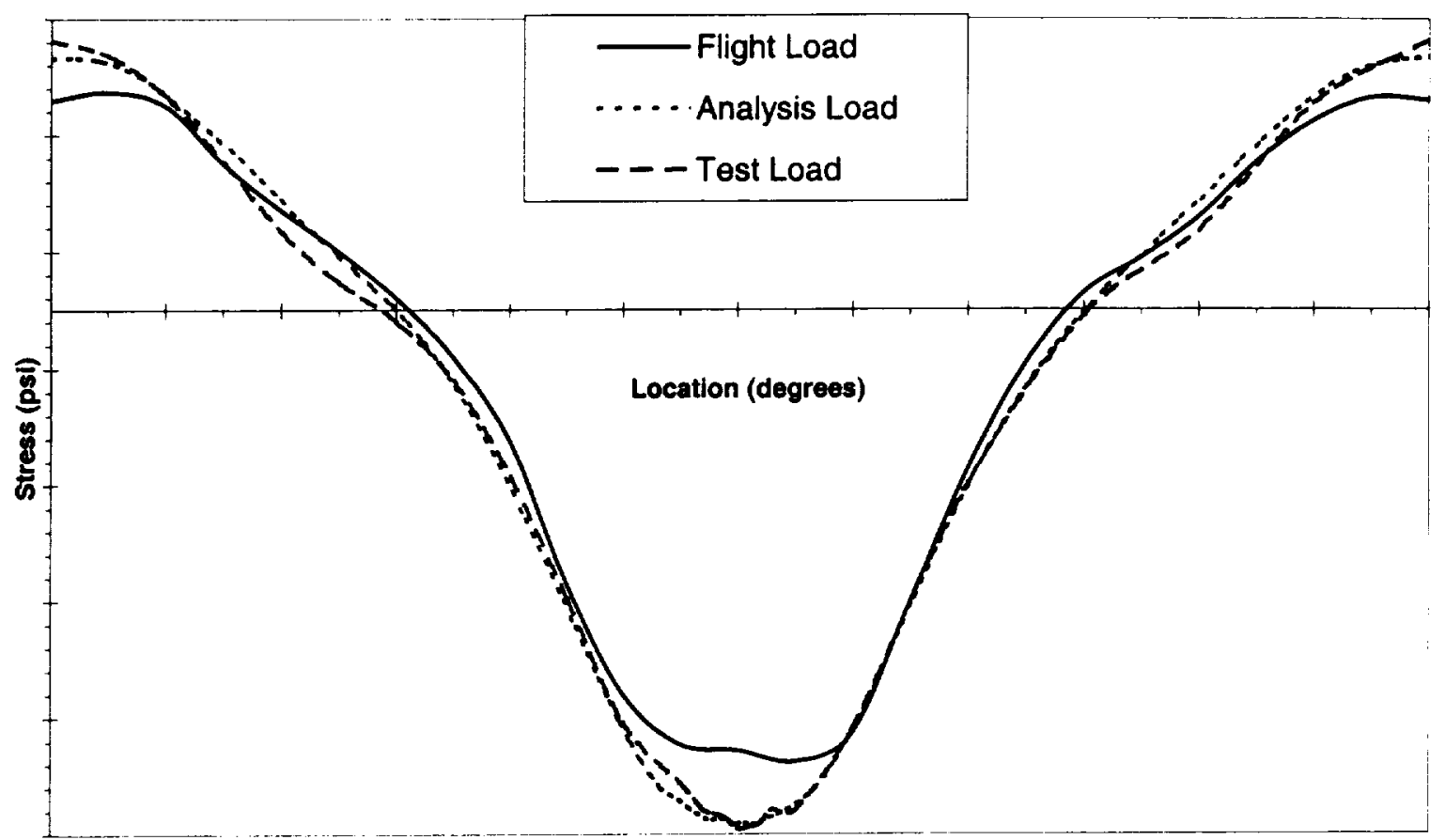

Figure 4 Line Load Comparisons

circumferential direction and is constant at any axial location. Therefore, the load at the compressive centerline would be constant along the entire length of the test article. Figure 4 shows the distribution of the axial stress around the circumference of a test article. It also shows the load distribution at the aft stiffener cylinder during pre-launch loading, and the analytically predicted load results. The test fixture was intentionally designed with this load distribution to facilitate the imperfection study and the overall testing effort. Since actual RSRM hardware was used in the testing, it would have been difficult and unlikely to find a test specimen with an imperfection located in the exact center of the stiffener center bay. The loading generated by the test fixture allowed the selection and study of several different stiffeners with a variety of imperfections. It also provided the means to study the complex behavior of multiple interacting imperfections without the influence of load variation.

Six stiffener cylinders were tested to determine the bounds of the RSRM buckling capability and to isolate the effects of several critical variables (eg. thickness and geometric imperfection (wall straightness)) on the buckling capability of RSRM stiffener cylinders (see Figure 5). Cylinders were selected from the flight inventory to isolate and evaluate the influence of these critical and controllable variables on RSRM buckling. Test cylinders were also selected to establish the limiting RSRM buckling capability based on the existing engineering requirements. An extensive inspection of the stiffener cylinder was performed prior to testing which provided a detailed map of case wall thickness and straightness. This data was critical in determining the orientation of the test article in the test fixture and providing data to create an accurate representation of the test article in the finite element model. Test articles were positioned in the load frame based on the test objective, the location and size of imperfection, and the case wall thickness. The six tests included cylinders with a nominal data point (nominal wall thickness and imperfection), two maximum imperfection tests, minimum wall thickness test, stiffener T-ring test, and a maximum thickness test.

Although only six tests were planned, it was discovered that after buckling occurred, the cylinder joints were 
TEST A T TICLES

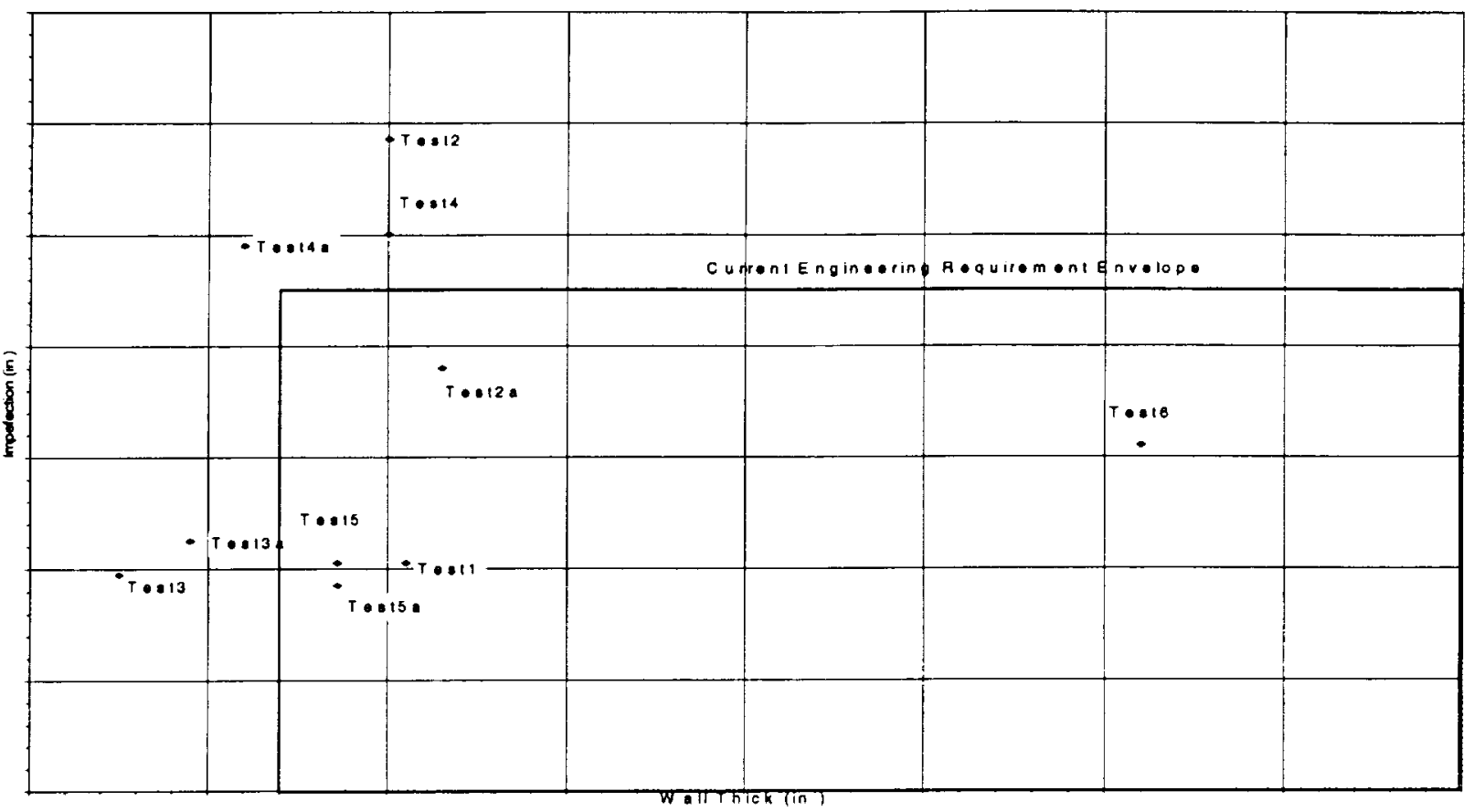

Figure 5 Test Article Selection

undamaged and cylinders could be rotated 180 degrees and tested again. Therefore, four additional tests were conducted to increase the amount of data collected from the testing.

The test cylinders as well as the fixture were instrumented to gather data to correlate with the finite element model. The instrumentation included strain gages to determine the load distribution around the circumference of the cylinder. Additional strain gages were positioned in regions where buckling was likely to occur (i.e. high imperfection locations and thin spots in the cylinder wall). Displacement of the actuators was measured as well as displacement of the case wall on the compressive and tensile sides. Actuator pressures to correlate the load input into the system were also measured. The test was video taped as well as photographed.

\section{Test Results}

The successful completion of testing provided new empirical data for full scale RSRM hardware. Prior to the testing, the influence of all critical buckling parameters was assumed to be the same as those of small diameter, thin wall cylinders. The most valuable result of the testing was the generation of data to provide a means to separate and evaluate the most critical variables with respect to RSRM buckling capability. The variables that had the greatest effect on the buckling capability were the material properties of the test cylinder, size of the imperfection, location of the imperfection, the shape of the imperfection, and the test cylinder wall thickness.

Each test specimen varied from the baseline in wall thickness, imperfection magnitude, and material properties. This made it difficult to separate and determine the influence of the critical variables. The approach used to separate the variables was to normalize all of the data for comparison by adjusting the maximum load. The testing indicated the ultimate buckling load was directly proportional to the elastic modulus of the material. Minor changes in Poisson's ratio did not appear to have a significant effect on the buckling capability. The magnitude of the imperfection had a linear relationship to the buckling failure load. A decrease in imperfection magnitude resulted in an increase in buckling capability. The imperfection magnitude had the greatest effect on buckling capability. The variation in wall thickness had the least significant influence of the critical variables. This was due to the small variation in RSRM case wall thickness. A larger variation in thickness would have had a larger influence on the buckling capability. The implementation of stiffener $T$-rings in the circumferential direction appeared to have some 
positive influence on the buckling capability of the cylinder.

As previously mentioned, there were different variables analyzed between tests. The variability in the test procedure was calculated by normalizing the data for material properties, thickness and imperfection. The resulting test variation was small when normalized to a given imperfection for an equivalent load, given thickness and material properties. The maximum variation of an estimate of the test error based on the established influence of thickness, imperfection, material property and load was $7.1 \%$.

The test instrumentation also yielded interesting results. Strain gages that were applied to the inside and outside diameter at the same location indicated a divergence in

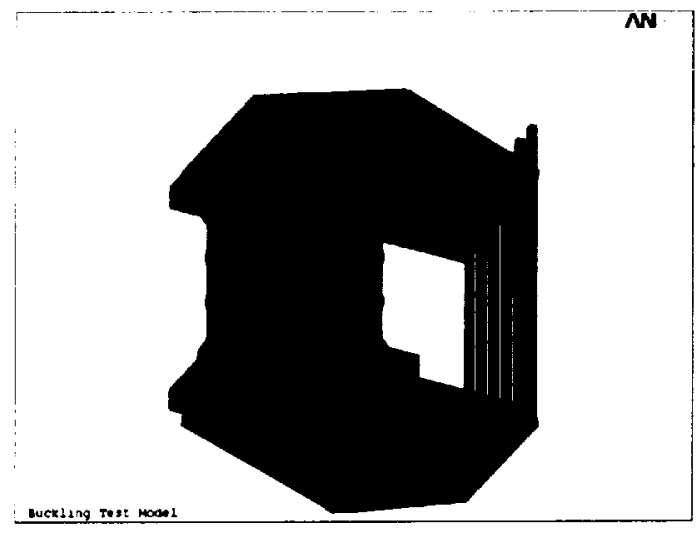

Figure 6 Nonlinear Finite Element Model

strain as the load increased to buckling failure. The divergence was not observed on all the gages, but was indicated where gages were placed at the critical failure location. It was observed that this phenomenon was not a totally reliable indicator of buckling failure but generally followed the failure pattern.

\section{Analysis}

A detailed finite element model using ANSYS53 finite element code was developed to simulate the loading of the test configuration (see Figure 6). All components of the test fixture were modeled, including the bolt configuration, to properly distribute the load into the test cylinder. Cylinder data was collected, which included wall thickness every 25 inches circumferentially and axially, and surface profile measurements every five inches circumferentially and axially. The data was used in the model to represent the actual geometry of the cylinder being tested.

The loading of the test model used a linear actuator element in ANSYS53 to simulate the actual actuators used in the testing. Symmetry boundary conditions were used at the midpoint of the model. Constraint equations held the force frame to the force ring, thus representing the bolt. Similarly, the buckling beams were attached to the force frame with constraint equations.

\section{Analysis/Test Correlation}

Buckling capability was predicted using both the eigenvalue analysis (with a knockdown factor) as well as nonlinear analysis. For each test, a comparison was made between the test results and the analytical prediction to develop and refine the nonlinear approach and determine the accuracy of the eigenvalue and nonlinear analyses.

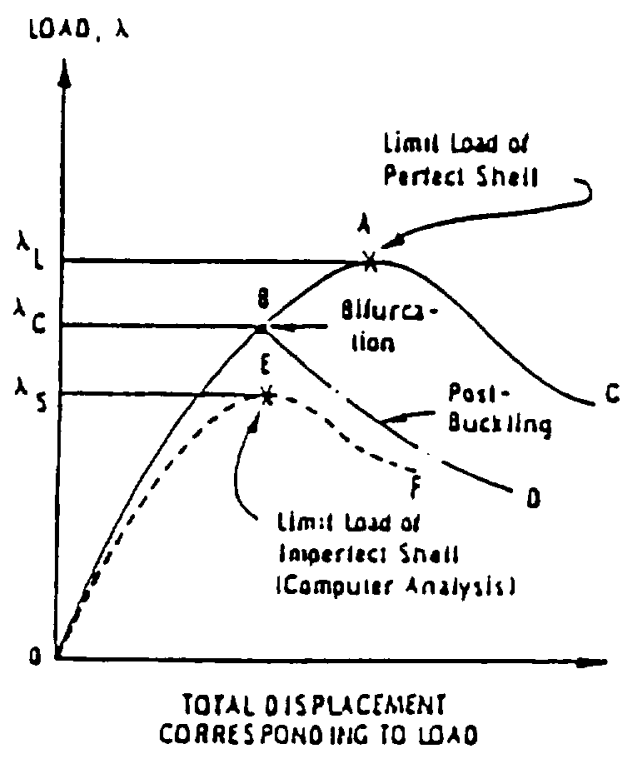

Figure 7 Eigenvalue Buckling Analysis

Eigenvalue buckling analysis predicts the theoretical buckling strength (bifurcation point) of a linear elastic analysis (see Figure 7). An eigenvalue analysis is performed resulting in a load multiplication factor or eigenvalue. The eigenvalue is a factor of the applied load that will cause buckling in a perfect structure. Imperfections and nonlinearities prevent structures 
from achieving the theoretical eigenvalue. The eigenvalue is modified to include anything that would cause the structure to behave differently than the perfect structure. Some of these factors are imperfections, material properties, geometric variation, propellant effects, etc. The KDF are used to modify the eigenvalue results to more closely represent actual capability. The KDF for each individual cylinder was derived based on the inspection data collected prior to the test and small scale cylindrical testing performed by

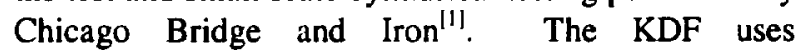
imperfection magnitude and size and wall thickness to determine its value from Equation 1.

$$
K D F=-0.0410-0.4693 * \log _{10}\left(\frac{U R}{t}\right) \quad \text { Eqn }
$$

Where :

$U=\frac{W_{o}}{4 R}$

$W_{o}=$ Im perfection Magnitude

$\mathrm{R}=$ Radius

$\mathrm{t}=$ Thickness

The KDF was applied to the eigenvalue solution, which was performed using ANSYS53 finite element code. The error between the eigenvalue solution with the knockdown factor and the buckling failure of the ten test specimens ranged from 9.5 to $27.4 \%$ (see Table 1). In all cases the actual test failure load was greater than the predicted failure using eigenvalue and $\mathrm{KDF}$.

Nonlinear buckling analysis is usually a more accurate analytical approach employing a nonlinear static analysis with gradually increasing loads to seek the load level at which the structure becomes unstable. Using this technique, features such as initial imperfection, wall thickness, joints and gaps and large deflection response are included. Prior to each test, a detailed finite element model of the test configuration was created using inspection data and actual geometry measurements.

Several factors in nonlinear analysis affect the final buckling capability of a structure. Each of these factors were studied using the results of the buckling test to determine the critical parameters to accurately predict the buckling capability of RSRM hardware.

The mesh resolution of the model was scaled to $2 \mathrm{X}$ and $4 \mathrm{X}$ to determine the critical mesh density for accurate buckling capability predictions. The final resolution resulted in elements measuring approximately four inches square. Finer meshing resulted in much longer computer run times with no noticeable increase in accuracy.

Performing a nonlinear buckling analysis using ANSYS53 finite element code offers the option of setting several convergence tolerance and solution controls. These included force and moment convergence tolerances as well as solution controls for line search, which aids in convergence and the number of equilibrium iterations. For the present work, the force and moment tolerances were very tight, line search was not useful, and the number of iterations for accuracy was ten.

Finite element modeling requires accurate load application to the structure. The simulation of the loading must, as closely as possible, represent the loading performed on the test. The actuators used in the test were closely modeled using a LINK11 element. Another critical modeling factor was adequate representation of part interface. The transfer of loading through different parts of the test and model must closely represent one another.

The hardware surface profile was critical in determining the buckling capability. The more accurate the measurements of the surface and the more accurate the surface was represented in the model, the more accurate the results. The influence of other imperfections near the critical buckling imperfection was also noted during the test. Therefore, the entire surface profile of the cylinder was used to determine the predicted buckling capability of the cylinder.

As the finite element analysis was performed, it was monitored and evaluated to verify proper behavior of the model with respect to the testing. The behavior of the model through displacement, loading, and strains must closely follow the results of the test. Without the testing, verification that an accurate finite element analysis was performed could not be achieved.

The results of the nonlinear analytical to test correlation indicate an improvement in buckling capability predictions over the eigenvalue analysis, as shown in Table 1. For the ten tests listed, the maximum error when using the nonlinear analysis method was $5 \%$. The method not only predicted the failure load accurately, it also identified the location and shape of the failure. The accuracy and repeatability of the correlation also indicated there was no need for a knockdown factor with nonlinear analysis. The test results shown in Table 
1 indicate the eigenvalue approach is conservative. Results also indicate there is sufficient conservatism to warrant an improved analytical approach. Furthermore, the test results were used to establish the critical buckling parameters, eliminating the uncertainty associated with the eigenvalue analysis.

\begin{tabular}{|c|c|c|c|}
\hline $\begin{array}{l}\text { Test } \\
\#\end{array}$ & Description & $\begin{array}{l}\text { \%Error } \\
\text { Nonlinear } \\
\text { Analysis }\end{array}$ & $\begin{array}{l}\text { \%Error } \\
\text { Eigenvalue } \\
\text { Analysis }\end{array}$ \\
\hline 1 & Baseline & 2.19 & 9.53 \\
\hline 2 & $\begin{array}{l}\text { Maximum } \\
\text { Imperfection }\end{array}$ & -0.57 & 27.01 \\
\hline $2 a$ & $\begin{array}{l}\text { Imperfection } \\
\text { Variation } \\
\text { Baseline } \\
\text { Comparison } \\
\end{array}$ & 1.32 & 24.02 \\
\hline 3 & $\begin{array}{l}\text { Minimum } \\
\text { Thickness }\end{array}$ & 1.95 & 26.01 \\
\hline $3 a$ & $\begin{array}{l}\text { Thickness } \\
\text { Variation }\end{array}$ & 1.62 & 17.75 \\
\hline 4 & $\begin{array}{l}\text { Minimum } \\
\text { Capability }\end{array}$ & 1.24 & 17.47 \\
\hline $4 a$ & $\begin{array}{l}\text { Variation } \\
\text { of Minimum } \\
\text { Capability } \\
\end{array}$ & 4.94 & 24.59 \\
\hline 5 & $\begin{array}{l}\text { Influence of } \\
\text { T-ring }\end{array}$ & 0.47 & 13.91 \\
\hline $5 a$ & $\begin{array}{l}\text { Influence of } \\
\mathrm{T} \text {-ring } \\
\text { Baseline } \\
\text { Comparison }\end{array}$ & 1.56 & 27.38 \\
\hline 6 & $\begin{array}{l}\text { Maximum } \\
\text { Capability }\end{array}$ & 4.18 & 10.03 \\
\hline
\end{tabular}

Table 1

\section{CONCLUSION}

Prior to this buckling test, neither large diameter cylinder nor full-scale RSRM hardware buckling test data existed. The buckling test indicates that previous eigenvalue analysis for large diameter, thin walled cylindrical shells is conservative, and that nonlinear analysis is much more accurate when properly applied. Several important concepts were determined as a result of the buckling tests, both from an analytical and hardware capability viewpoint, that are critical to accurate prediction of buckling capability using nonlinear analysis.
1) The mesh resolution can limit the accuracy of the analysis and the calculated failure mode.

2) Convergence tolerance and solution controls must be refined and verified by testing.

3) Proper load application in the model is critical to the accuracy and assessment of the buckling capability,

4) Adequate representation of part interfaces can significantly influence results.

5) Slight changes in hardware surface profile can result in a large change in buckling capability.

6) Care must be taken to monitor and evaluate the analysis as it is progressing to verify proper model behavior through expected load increment and displacement.

Nonlinear finite element analysis accurately predicts buckling capability with the application of test result concepts to the structure. Results of this testing and analysis work were subsequently applied to a full scale RSRM model to predict the pre-launch buckling capability of the space shuttle system, thus removing over-conservative wind restraints.

\section{Reference:}

1. Miller, C.D., "Buckling Stresses for Axially Compressed Cylinders", Chicago Bridge and Iron Company Report, CBT 5394, August 1976. 